\title{
G

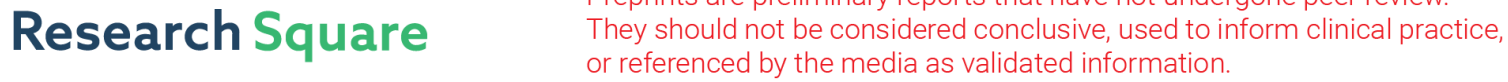 \\ Effects of Refractive Accommodation on Subfoveal Choroidal Thickness in Silicone Oil-Filled Eyes
}

\section{Ying Yan}

PLA Middle Theater General Hospital

\section{Ran Liu}

PLA Middle Theater General Hospital

\section{Chengyuan Gao}

PLA Middle Theater General Hospital

\section{Yanping Song}

PLA Middle Theater General Hospital

\section{Qin Ding}

PLA Middle Theater General Hospital

\section{Feng Chang}

PLA Middle Theater General Hospital

Xiao Chen ( $\square$ cxfn817@163.com )

PLA Middle Theater General Hospital

\section{Research Article}

Keywords: choroidal vascularity index, optical coherence tomography, silicone oil, subfoveal choroidal thickness.

Posted Date: August 17th, 2021

DOI: https://doi.org/10.21203/rs.3.rs-805747/v1

License: (c) (i) This work is licensed under a Creative Commons Attribution 4.0 International License. Read Full License

Version of Record: A version of this preprint was published at BMC Ophthalmology on March 5th, 2022. See the published version at https://doi.org/10.1186/s12886-022-02332-y. 


\section{Abstract}

PURPOSE: To investigate the effects of refractive accommodation on subfoveal choroidal thickness (SFCT) in silicone oil (SO)-filled eyes.

METHODS: This retrospective, self-comparative study was conducted on 40 patients with unilateral macula-on rhegmatogenous retinal detachment, who underwent vitrectomy and SO tamponade. The SFCT of SO-filled eyes and the fellow control eyes were measured using optical coherence tomography at their one-month visit after surgery. The patients wore soft contact positive lenses for 24 hours in the SOfilled eyes, to correct their refractive error. SFCT and choroidal vascularity index (CVI) were measured before and after wearing the contact lenses. Mean SFCT was compared between SO-filled eyes and the fellow control eyes, and SFCT and CVI were compared before and after refractive error correction in the so-filled eyes.

RESULTS: Mean SFCT of SO-filled eyes (221.52 $\pm 38.41 \mathrm{um})$ was less than that of the fellow eyes $(273.41 \pm 31.30 \mathrm{um})(\mathrm{P}<0.001)$. After refractive error correction, the mean SFCT increased to $269.28 \pm 36.90$ um $(P<0.001)$. However, $C V I$ decreased from $57.01 \pm 2.41$ to $55.39 \pm 2.39(P<0.05)$.

CONCLUSION: SFCT reduction in SO-filled eyes was primarily due to the hyperopia status. The nonuniform change in CVI suggests that changes in CT are mainly attributed to a greater expansion of the stromal area instead of the choroidal vascular area.

\section{Introduction}

Silicone oil (SO) has been used as an intraocular tamponade, since many years in vitreoretinal surgery, and its toxic effects on retina have been reported through several studies.1-4 Some recent studies have reported that SO leads to a reduction in choroidal thickness (CT),5-6 which can also be considered a toxic effect on choroidal tissues.7-8 However, SO induced refractive error (RE) (usually present in hyperopia), which can considerably influence the CT value, has not been adequately evaluated in these studies. As the refractive state could have a substantial impact on CT,9-11 we hypothesized that the RE induced by SO tamponade has an impact on the CT.

In the present study, we measured the SFCT of the SO-filled eyes and fellow control eyes and compared the changes in SFCT after the correction of RE in the SO-filled eyes. In addition, we analyzed choroidal vascularity index $(\mathrm{CVI})$ that seems to be a robust marker12 to assess choroidal vascular status in these patients.

\section{Materials And Methods}

\section{Patients}


This retrospective, interventional comparative study was conducted on 40 patients with unilateral macula-on rhegmatogenous retinal detachment, recruited from the Department of Ophthalmology at Central Theater General Hospital. The fellow 40 eyes were served as control. The patients were successfully treated with a three-port pars plana vitrectomy (PPV) and a 5,700-cSt SO endotamponade (Bausch\&Lomb, New York,United States of America).

\section{Exclusion :}

1. Patients with documented previous ocular disease (glaucoma, diabetic retinopathy, macular degeneration

2. high myopia exceeding -6.0 diopters

3. with other than uncomplicated cataract surgery

4. Macular-off RRD

5. Received more than one surgery repaired

6. With PVR grade 3 or 4 and eceived retinotomy

7. with choroidal detachment

8. Intraocular pressure greater than $21 \mathrm{mmHg}$

9. Considering intraocular pressure[13] and antiglaucomatous drops[14] had a significant effect on SFCT, we excluded the patients postoperative intraocular pressure more than $21 \mathrm{mmHg}$.

This study was approved by the institutional review board at Central Theater General Hospital and was conducted in accordance with the Declaration of Helsinki. All the patients were informed of the nature of their disease and of all potential treatment options, and informed consents were obtained.

A complete ocular examination was conducted in each patient, including the determination of bestcorrected visual acuity (BCVA) using standard Snellen eye charts, RE screened with autorefractor (Canon Autorefractor RK-F1, Canon Inc. Ltd., Tochigiken, Japan), measurement of intraocular pressure (IOP), slitlamp examination, stereoscopic observation of the fundus, and macular measurements using sweptsource optical coherence tomography (SS-OCT) (DRI OCT-1 Atlantis, TOPCON Corp, Tokyo, Japan).

Optical Coherence Tomography Measurements

SFCT was measured using SS-OCT. According to the guidelines suggested by Kikushima, 15 the quality of OCT images was automatically evaluated by Topcon image quality factor, and scored from 0 to 100 . Only high-quality images (quality factor $\geq 60$ ) were used for analysis.

CT was defined as the distance between the base of the subfoveal retinal pigment epithelium and the margin of the choroidoscleral interface. SFCT was measured at the fovea, and at 500-um distances up to $1.5 \mathrm{~mm}$, temporal and nasal to the fovea in horizontal direction, and up to $1.5 \mathrm{~mm}$, superior and inferior to the fovea in vertical direction using the manual segmentation method (shown in Fig. 1). The mean overall SFCT was obtained by calculating the average value of SFCT measurements at all eccentricities. 
$\mathrm{CVI}$, defined as the proportion of vascular area to the total choroidal area, was measured on the SS-OCT images.16-17 In brief, after uploading the images on the ImageJ software (http://imagej.nih.gov/ij/; provided in the public domain by the National Institute of Health), they were converted to 8-bit images to allow application of the autothreshold. A 3000-um thick line centered at the fovea was drawn. The part of choroid beneath the line was segmented, and total subfoveal choroidal area was computed. The auto local threshold was determined using the Niblack auto local threshold to demarcate the choroidal vascular area and stromal area (shown in Fig. 2).

The OCT scans were performed in both eyes to measure SFCT and CVI, one month after surgery. The SOfilled eyes wore soft, positive, contact lenses (Kushi, China) for 24 hours to correct the RE, depending on its optometry value. SFCT and CVI were measured once again, before removing the contact lenses. Mean SFCT and CVI were compared before and after wearing lenses in SO-filled eyes. Considering the diurnal variation in $\mathrm{CT}$, all examinations were performed between 9 a.m. and 10 a.m.

The measurements were performed manually by two retina specialists using calipers provided with the device, and the averaged values were saved and considered for statistical analysis.

Statistical Analyses

All statistical analyses were performed with a commercial analytical package (SPSS Statistics 22.0 for Windows; SPSS, Inc., IBM, Somers, NY, USA). Continuous variables were expressed as mean \pm standard deviation. Paired $t$-tests and unpaired t-tests were used to analyze numerical and ordinal variables, respectively. A P value $<0.05$ was considered to be statistically significant.

\section{Results}

Twenty-five men and fifteen women (mean age, 38.47 \pm 8.02 years; range, 20-50 years) were enrolled. Anatomical success (complete retinal reattachment) was noted in all cases. Mean RE of the SO-filled eyes was $+6.64 \pm 1.25 \mathrm{D}$, ranging from +4.5 to $+9.5 \mathrm{D}$. Mean preoperative BCVA was $20 / 125$, ranging from $20 / 200-20 / 50$ (logarithm of the minimum angle of resolution (logMAR) was $0.81 \pm 0.28$ ).

The mean BCVA 1 month after surgery was 20/63, ranging from 20/100 - 20/25 (logMAR was $0.49 \pm$ $0.22)(P<0.001)$. The mean preoperative IOP was $12.3 \pm 4.2 \mathrm{mmHg}$, and mean IOP 1 month after surgery was $16.4 \pm 3.5 \mathrm{mmHg}(P>0.05)$. Demographic and clinical characteristics of patients are summarized in Table 1.

It was observed that the mean SFCT of SO-filled eyes was $221.52 \pm 38.41$ um, mean SFCT of the fellow eyes was $273.41 \pm 31.30 \mathrm{um}$, and SFCT of SO-filled eyes was significantly less than that of the fellow eyes, 1 month after SO tamponade $(P<0.001)$. Mean SFCT of SO-tamponaded eyes after wearing lenses was $269.28 \pm 36.90$ um. Mean SFCT of SO-tamponaded eyes significantly increased by wearing positive contact lenses $(P<0.001)$ (shown in Fig. 3).

However, CVI decreased from $57.01 \pm 2.41$ to $55.39 \pm 2.39(\mathrm{P}=0.044)$ after RE correction. 


\section{Discussion}

In this study, we observed that SFCT of SO-filled eyes was significantly less than that of the fellow control eyes, 1 month after vitrectomy and SO tamponade. Similar results were obtained by Odroniba et al. and Karimi et al.,18-19 who reported that SFCT decreased after PPV and SO tamponade. Sugawara et al.20 showed that CT of eyes with retinal detachment was similar to the fellow eyes before treatment. Therefore, previous studies have shown that PPV, by itself, did not have any significant effect on CT.21 These findings suggest that the reduction in SFCT of SO-filled eyes after PPV might be related to the negative effects of intravitreal SO.

However, the possible mechanism of SO tamponade leading to a decrease in choroid thickness is not clearly understood. Some authors have proposed that SO toxicity might be caused by a failure of potassium siphoning by the Müller cells.22Another mechanism by Odrobina D suggested that SO could induce a type of microangiopathy in the retina and choroid, leading to altered blood flow and ischemia, subsequently causing retinal and choroidal thinning. 18 However, the mechanism has not been proved scientifically.

As early as in 1995, an interesting study performed on chickens by Wallman et al.9-10 showed that spectacle lens-induced hyperopia and myopia mediated the changes in thickness of the choroid. The choroid can increase its thickness in response to myopic defocus (image focused in front of the retina) by pushing the retina towards the image plane. On the contrary, choroidal thinning is observed in response to hyperopic defocus (image focused behind the retina).23 A similar choroidal mechanism for regulation of the refractive state has been proved to exist in humans as well.11

Based on these observations and the results of our study, we believe that the reduction in SFCT of SOfilled eyes moved the retina backward, bringing the photoreceptors into plane of focus. In contrast, increased SFCT can occur due to the backward movement of the focal plane by wearing positive lenses. To the best of our knowledge, this is the first study to investigated the effects of refractive accommodation on SFCT in SO-filled eyes.Results indicated SFCT reduction in SO-filled eyes was primarily due to the hyperopia status.

The choroid is a vascular layer that supplies oxygen and nutrients to the outer part of retina.24 Choroidal thinning may lead to a relative decrease in choroidal circulation, eventually leading to a reduced level of necessary oxygen and nutrient delivery to the most metabolically active foveal region. 25

In contrast to CT, CVI, defined as the proportion of the luminal area to the total choroid area, 26 shows lesser variability, being influenced by fewer physiological factors such as axial length, RE, intraocular pressure, systolic blood pressure, and diurnal variation.27lt can help in understanding the variations in vascularity of chorioretinal compartments through simultaneously obtained images by OCT.28

In our study, CVI of SO-filled eyes decreased after RE. After thoroughly analyzing our results, we concluded that the increase in thickness could be attributed to an expansion of the stromal area instead 
of choroidal vascular area. The theory is supported by a research conducted by Wallman, which stated that the oblique or tangential arrangement of the non-vascular smooth muscles between the lacunae makes it plausible that they might play a role in the changes in CT occurring in response to retinal defocus. 29

The principal limitations of this study include the relatively small sample size. SFCT and CVI were measured just 1 month after PPV. Nonetheless, the results can help in understanding the influence of hyperopia caused by SO on CT. In future, long-term prospective studies, involving a larger population, and a longer follow-up would be needed to improve our clinical understanding of CT and vascularity of choroid.

In conclusion, the reduction in SFCT of SO-filled eyes may be primarily due to the hyperopia caused by SO, which can be reversed after RE correction. The non-uniform change in CVI suggests that changes in CT are mainly attributed to an expansion of the stromal area instead of choroidal vascular area.

\section{Declarations}

\section{Statement of Ethics}

Study approval statement: This study protocol was reviewed and approved by the Ethics Committee of the Central Theater General Hospital, approval number No. [2020]058-1.

Consent to participate statement: Written informed consent was obtained from participants to participate in the study.

\section{Conflict of Interest Statement}

The authors have no conflicts of interest to declare.

\section{Funding Sources}

\section{The project of Natural Science Foundation of Hubei Province(ID 1403)}

\section{Author Contributions}

Xiao Chen performed the vitrectomy surgery ; Ying Yan and Ran Liu contributed significantly to analysis and manuscript preparation; Chengyuan Gao performed the data analyses; Qin Ding performed OCT examination; Yanping Song, Feng Chang helped perform the analysis with constructive discussions.

\section{Data Availability Statement}

The data that support the findings of this study are not publicly available due to their containing information that could compromise the privacy of research participants, but are available from Xiao Chen(cxfn817@163.com) upon reasonable request. 


\section{References}

1 Foulks G, Hatchell D, Proia A, Klintworth G. Histopathology of silicone oil keratopathy in humans. Cornea. 1991;10:29-37.

2 Honavar S, Goyal M, Majji A, Sen P, Naduvilath T, Dandona L. Glaucoma after pars plana vitrectomy and silicone oil injection for complicated retinal detachments. Ophthalmology. 1999;106:169-177.

3 Budde M, Cursiefen C, Holbach LM, Naumann GO. Silicone oil-associated optic nerve degeneration. Am J Ophthalmol. 2001;131:392-394.

4 Wickham L, Asaria RH, Alexander R, Luthert P, Charteris DG. Immunopathology of intraocular silicone oil: enucleated eyes. Br J Ophthalmol. 2007;91:253-257.

5 Odrobina D, Golebiewska J, Maroszynska I. Choroidal thickness changes after vitrectomy with silicone oiltamponade for proliferative vitreoretinopathy retinal detachment.Retina. 2017;37:2124-2129.

6 Karimi S, Entezari M, Nikkhah H, Esfandiari H, Darvishpoor T, Tavakoli M, et al. Effects of Intravitreal Silicone Oil on Subfoveal Choroidal Thickness. Ophthalmologica. 2018;239:159-166.

7 Odrobina D, Golebiewska J, Maroszynska I. Choroidal thickness changes after vitrectomy with silicone oil tamponade for proliferative vitreoretinopathy retinal detachment. Retina. 2017;37:2124-2129.

8 Winter M, Eberhardt W, Scholz C, Reichenbach A. Failure of potassium siphoning by Muller cells: a new hypothesis of perfluorocarbon liquid-induced retinopathy. Invest Ophthalmol Vis Sci. 2000;41:256-261.

9 Wallman J, Wildsoet C, Xu A, Gottlieb MD, Nickla DL, Marran L, et al. Moving the retina: choroidal modulation of refractive state. Vision Res. 1995;35:37-50.

10 Wildsoet C, Wallman J. Choroidal and scleral mechanisms of compensation for spectacle lenses in chicks. Vision Res. 1995;35: 1175-1194.

11 Poukens V, Glasgow BJ, Demer JL. Nonvascular Contractile Cells in Sclera and Choroid of Humans and Monkeys. Invest Ophthalmol Vis Sci. 1998;39:1765-1774

12 Agrawal R, Gupta P, Tan KA, Cheung CM, Wong TY, Cheng CY. Choroidal vascularity index as a measure of vascular status of the choroid: Measurements in healthy eyes from a populationbased study. Sci Rep. 2016;6:21090.

13 Kim M, Kim SS, Kwon HJ, Koh HJ, Lee SC. Association between Choroidal Thickness and Ocular Perfusion Pressure in Young, Healthy Subjects: Enhanced Depth Imaging Optical Coherence Tomography Study. Invest Ophthalmol Vis Sci. 2012;53:7710-7717.

14 Okutucu M, Fındık H, Arslan MG. Direct and crossover effects of brinzolamide, betaxolol, and latanoprost on choroidal thickness. Cutan Ocul Toxicol. 2019 Jun;38(2):196-200. 
15 Kikushima W, Imai A, Toriyama Y, Hirano T, Murata T, Ishibashi T. Dynamics of macular hole closure in gas-fifilled eyes within $24 \mathrm{~h}$ of surgery observed with swept source optical coherence tomography. Ophthalmic Res. 2015;53:48-54.

16 Agrawal R, Salman M, Tan KA, Karampelas M, Sim DA, Keane PA, et al. Choroidal vascularity index (CVI)-a novel optical coherence tomography parameter for monitoring patients with panuveitis? PLoS One. 2016; $11: \mathrm{e} 0146344$.

17 Sonoda S, Sakamoto T, Yamashita T, Shirasawa M, Uchino E, Terasaki H, et al. Choroidal structure in normal eyes and after photodynamic therapy determined by binarization of optical coherence tomographic images. Invest Ophthalmol Vis Sci. 2014;55:3893-3899.

18 Odrobina D, Golebiewska J, Maroszynska I. Choroidal thickness changes after vitrectomy with silicone oil tamponade for proliferative vitreoretinopathy retinal detachment. Retina. 2017;37:2124-2129.

19 Karimi S, Entezari M, Nikkhah H, Esfandiari H, Darvishpoor T, Tavakoli M, et al. Effects of Intravitreal Silicone Oil on Subfoveal Choroidal Thickness. Retina. 2016;1-6.

20 Sugawara R, Nagaoka T, Kitaya N, Fujio N, Takahashi J, Takahashi A, et al. Choroidal blood flow in the foveal region in eyes with rhegmatogenous retinal detachment and scleral buckling procedures. $\mathrm{Br} \mathrm{J}$ Ophthalmol. 2006;90:1363-1365.

21 Sayman Muslubas I, Karacorlu M, Hocaoglu M, Arf S, Uysal O. Subfoveal choroidal thickness changes after pars plana vitrectomy in recent onset rhegmatogenous retinal detachment. Retina. 2016;36:23712376.

22 Winter M, Eberhardt W, Scholz C, Reichenbach A. Failure of potassium siphoning by Muller cells: a new hypothesis of perfluorocarbon liquid-induced retinopathy. Invest Ophthalmol Vis Sci. 2000;41:256-261.

23 Wallman J, Wildsoet C, Xu A, Gottlieb MD, Nickla DL, Marran L, et al. Moving the retina: choroidal modulation of refractive state. Vis. Res. 1995; 35;37-50.

24 Nickla DL, Wallman J. The multifunctional choroid. Prog Retin Eye Res. 2010;29:144-168.

25 Nishida Y, Fujiwara T, Imamura Y, Lima LH, Kurosaka D, Spaide RF. Choroidal thickness and visual acuity in highly myopic eyes. Retina. 2012;32:1229-1236.

26 Agrawal R, Gupta P, Tan KA, Cheung CM, Wong TY, Cheng CY. Choroidal vascularity index as a measure of vascular status of the choroid: Measurements in healthy eyes from a populationbased study. Sci Rep. 2016;6:21090.

27 Sansom LT, Suter CA, McKibbin M. The association between systolic blood pressure, ocular perfusion pressure and subfoveal choroidal thickness in normal individuals. Acta Ophthalmol. 2016;94(2):e157e158. 
28 Giannaccare G, Pellegrini M, Sebastiani S, Bernabei F, Moscardelli F, lovino C, et al. Choroidal Vascularity Index Quantification in Geographic Atrophy Using Binarization of Enhanced Depth Imaging Optical Coherence Tomographic Scans. Retina 2019. DOI: 10.1097/IAE.0000000000002459. [Epub ahead of print]

29 Junghans BM, Crewther SG, Liang H, Crewther DP. A role for choroidal lymphatics during recovery from form deprivation myopia? Optom. Vis. Sci. 1999;76;796-803.

\section{Tables}

Due to technical limitations, table 1 is only available as a download in the Supplemental Files section.

\section{Figures}

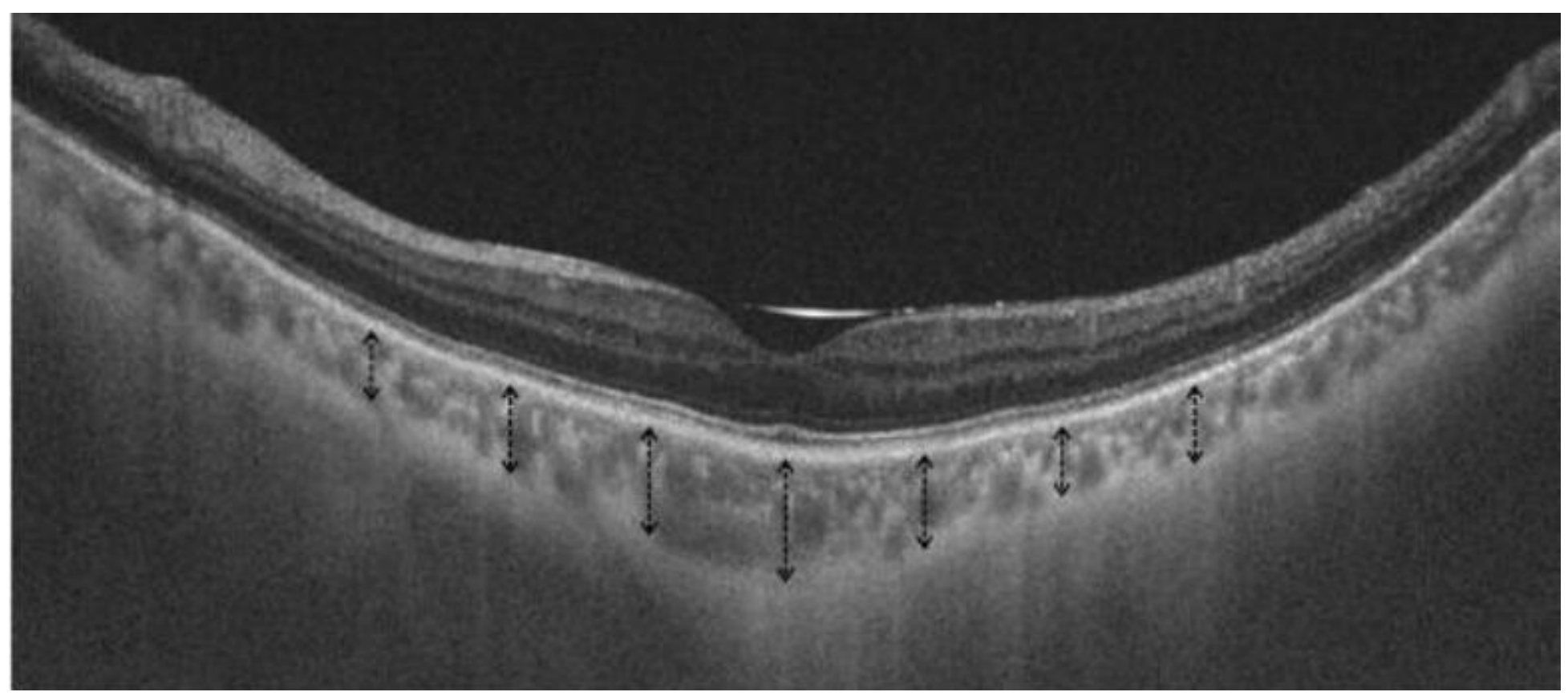

\section{Figure 1}

A representative SS-OCT measurement of SFCT. Black dashed arrows indicate an interval of $0.5 \mathrm{~mm}$ from the fovea up to $1.5 \mathrm{~mm}$ choroidal thickness. 


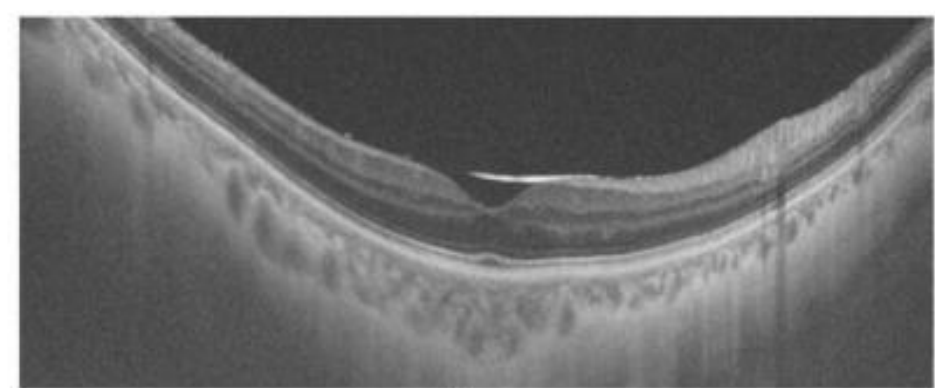

A

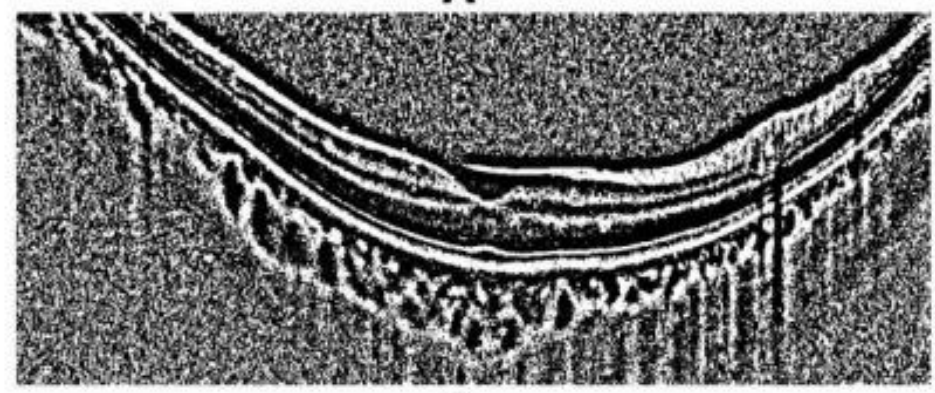

C

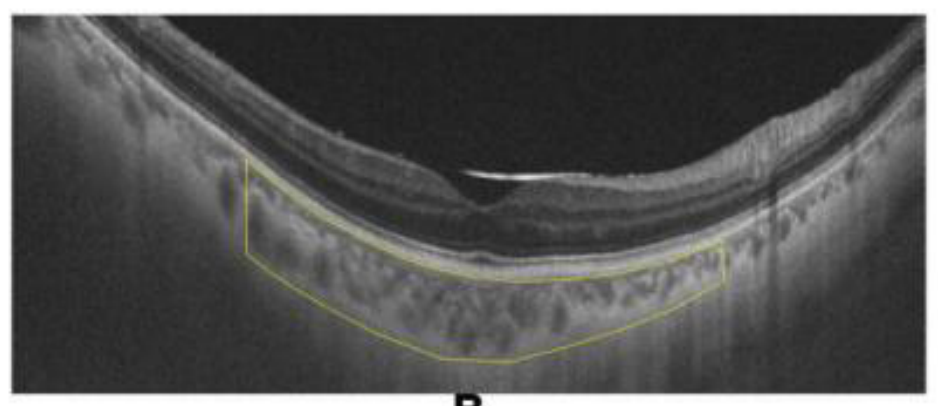

B

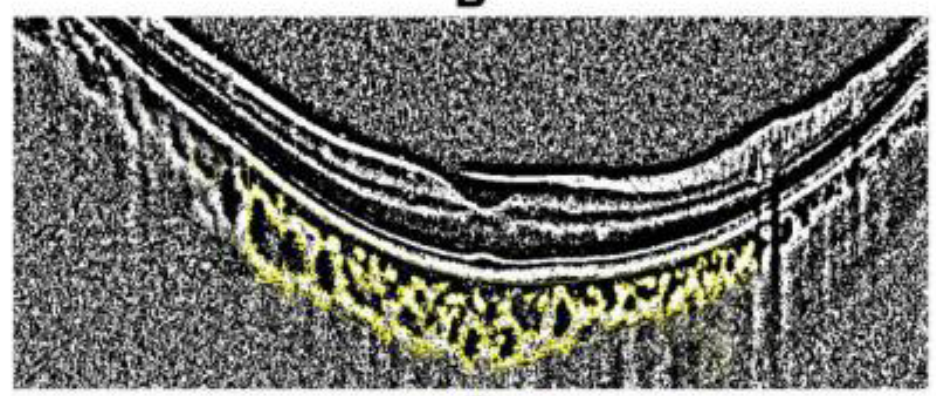

D

Figure 2

A Representative image processing to obtain $\mathrm{CVI}$ and choroidal vascular area. (A) Original SS-OCT image. (B) A 1.5-mm segmentation block of the subfoveal choroidal area using the polygon selection tool. (C) Segmented OCT image using a modifified image binarization approach. (D) The vascular area was highlighted by applying the color threshold. The ratio of the vascular area to the total choroidal area was termed the CVI.
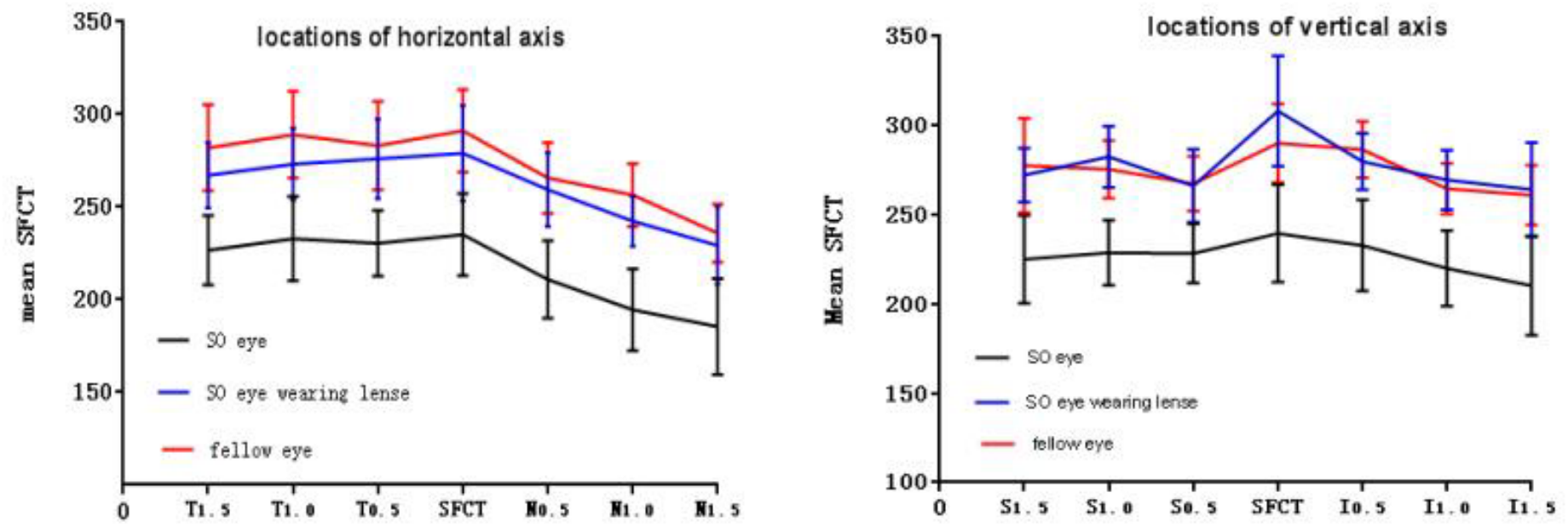

Figure 3

Graph showing the differences in three groups (followed eyes, SO tamponaded eyes before and after lenses wore) along the horizontal axis and vertical axis.

\section{Supplementary Files}


This is a list of supplementary files associated with this preprint. Click to download.

- Table1.pdf 